\title{
Influence of sistemic osteoporosis on periodontal health: epigenetic micro-RNA resemblances
}

\author{
Dan Piperea-Sianu', StefanCristea ${ }^{2}$ \\ ${ }^{1}$ Physiology Department, Faculty of Dental Medicine, Bucharest, Romania \\ ${ }^{2}$ Orthopaedics and Traumatology Department, Faculty of General Medicine, \\ "Carol Davila" University of Medicine and Pharmacy, Bucharest, Romania
}

\begin{abstract}
Osteoporosis and periodontal disease are two diseases that affect millions of people worldwide. The common element is represented by bone loss. Periodontal disease consists of a set of inflammatory and/or degenerative changes concerning the periodontium, that evolve in the first phase with the loss of the connection between the superficial periodontium and the tooth, and subsequently with the damage of the profound periodontium. Recent studies in the literature show that osteoporosis, by lowering systemic bone mineral density and alveolar bone, can create a favorable framework for the progression of the periodontal impairment. This paper aims to present the main epigenetic changes related to micro-RNA from the two pathologies, focusing on the common elements that they share.
\end{abstract}

Keywords: osteoporosis, periodontal disease, epigenetics

\section{INTRODUCTION}

The genetic factor is essential in determining the maximum bone mineral density (BMD) possible, but also the bone structure. The genotype may predispose to damage, frailty and fractures. However, the genetic factor can not, by itself, be responsible for the occurrence of osteoporosis or periodontal disease. Long-term interactions between genetic factors and environmental factors are required so that the anatomical changes happen (1).

The synergistic action of environmental factors with genetic factors may be a triggering factor in osteoporotic pathology. Understanding epigenetic mechanisms is essential in order to improve understanding of the etiopathogenicity of the disease. The complete understanding of the pathogenesis of a disease is an ideal that once achieved, helps to develop clinical strategies to prevent disease, complications, and also new therapeutic methods (1).

In a normal adult, the bone remodeling process consists of a balanced association of two processes called resorption and bone apposition. The balance between these processes requires strict control of osteoblastic (apposition) and osteoclastic (resorption) activity. Osteoblasts and osteoclasts are under genetic control, which determines bone phenotype and mineral density. It is important to note that the polymorphism of the genes involved in this control is responsible for approximately $10 \%$ of the phenotypic variation in bone density $(1,2)$.

Epigenetic mechanisms consist of changes in the expression of proteins and genes, independently of the inherited DNA nucleotide sequence. These mechanisms include histone changes, methylation of deoxyribonucleic acid, and negative modulation of messenger RNA mediated by post-transcriptional micro-RNA chains.

\section{Noncoding RNA in the pathogenesis of osteoporosis}

Noncoding RNA (ncRNA) represents approximately $98 \%$ of the total RNA that is formed from DNA transcription. Only $2 \%$ of RNA is used for protein synthesis, the remaining $98 \%$ is not used for this purpose and constitutes the non-coding RNA. It participates in regulating gene expression by controlling transcription and post-transcription processes (3-6). 
Non-coding RNA is classified in infrastructural RNA and regulatory RNA. Non-coding RNA with epigenetic role is the regulatory RNA (3).

Another classification of non-coding RNA and the roles of each type is: (a) micro-RNA (Fig. 1), consists of a single RNA strand and plays a role in gene inhibition; (b) pi-RNA, has a role in inhibiting transposable elements; (c) si-RNA, consists of two RNA strands and plays a role in post-transcriptional inhibition and antiviral protection; (d) pa-RNA, consists of a single RNA strand with reduced half-life and plays a role in post-transcriptional modulation; (e) eRNA, consists of a single RNA chain with reduced half-life and plays a role in transcriptional gene activation; (f) lnc-RNA: represents the noncoding RNA with long molecule and plays a role in transcriptional, post-transcriptional modulation, and siRNA precursors modulation (3).

Lnc-RNA is recently considered antagonist differentiating noncoding RNA and has been shown to have the following roles $(3,7,8)$ :

- mediation of osteoblastic differentiation;

- its individual overexpression inhibits osteoblastic differentiation;

- association with si-RNA favors osteoblastic differentiation;

- stimulates the formation of IL-6, TNF- $\alpha$;

- its expression is directly proportional with the serum concentration of IL-6 in women postmenopause.

Of all the noncoding RNA subtypes, the most studied in relation to bone resorption-apposition balance is mi-RNA. Changes in mi-RNA expression may cause disorders in bone metabolism. From this point of view, mi-RNA is divided into two categories according to Ghayor $\mathrm{C}$ and Weber $\mathrm{F}$ :
- osteoblastic differentiation modulators: stimulating $(m i R-15 b, m i R-21, m i R-27, m i R-29 a, m i R-96$, miR-128, miR-142-3, pmiR-194, miR-216-a, miR335-5p, miR-2861, miR-3960), inhibitori (Lnc-ARN, miR-23-a, miR-124, miR-133, miR-135, miR-153, miR-204, miR-223, miR-375);

- osteoclastic differentiation modulators: stimulating (Lnc-ARN, miR-21, miR-34-a, miR-183, miR214, miR-223, miR-9718), inhibitori (miR-7-b, miR17/20a, miR-26-a, miR-126, miR-155).

Osteoclasts are bone marrow cells that come from macrophages. The most important step in osteoclastic differentiation is the fusion of mononuclear precursors to form multinuclear cells. An important factor that promotes macrophage fusion and differentiation is DCSTAMP (specific transmembrane protein of the dendritic cells). miR-7b inhibits DCSTAMP and thus inhibits cell fusion and osteoclastogenesis. miR-26a inhibits CTGF (connective tissue growth factor), which plays a role in stimulating DCSTAMP. Overexpression of miR-26a inhibitors favors osteoclast formation via dependent RANK-L pathway (3,9-11).

Micro-RNA may influence osteogenic differentiation. In osteoporotic patients it has been observed that there is an overexpression of some types of miRNA. Resveratrol, a polyphenolic phytoestrogen, proves to have the ability to inhibit miR-338-3p. By inhibiting miR-338-3p, resveratrol stimulates osteogenic differentiation and has osteoinductive capabilities. Inhibition of miR-338-3p is associated with RUNX2 stimulation. Also, Khazaei et al., Ribeiro et $a l$. and Zupancic et al. mention the role of resveratrol in limiting alveolar bone loss, improving clinical indices for periodontal evaluation, and improving periodontal bone osteoimmunoinflammatory markers $(3,12-16)$.

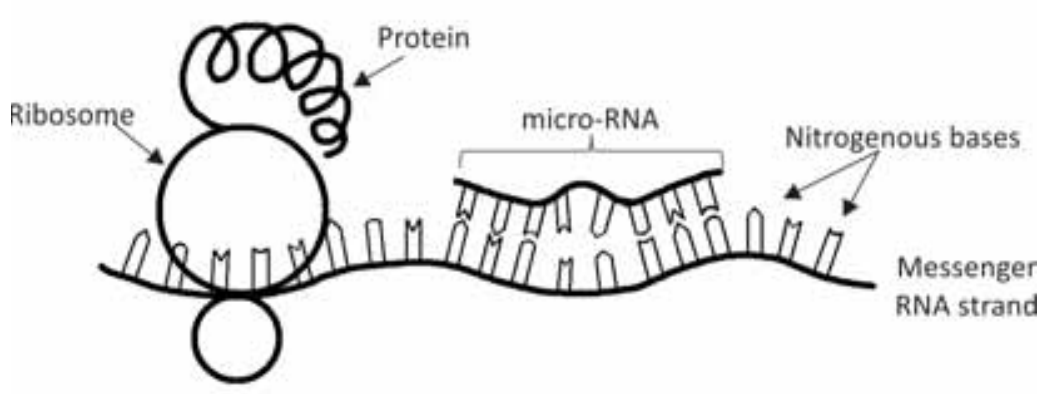

FIGURE 1. Inhibition of gene translation by micro-RNA.

The micro-RNA strand partially couples to messenger RNA nucleotides and inhibits the translation, synthesis of polypeptide chains and proteins. Ribosomes are depicted moving from left to right, reading the nucleotide sequence of the messenger RNA chain up to the micro-RNA level. 


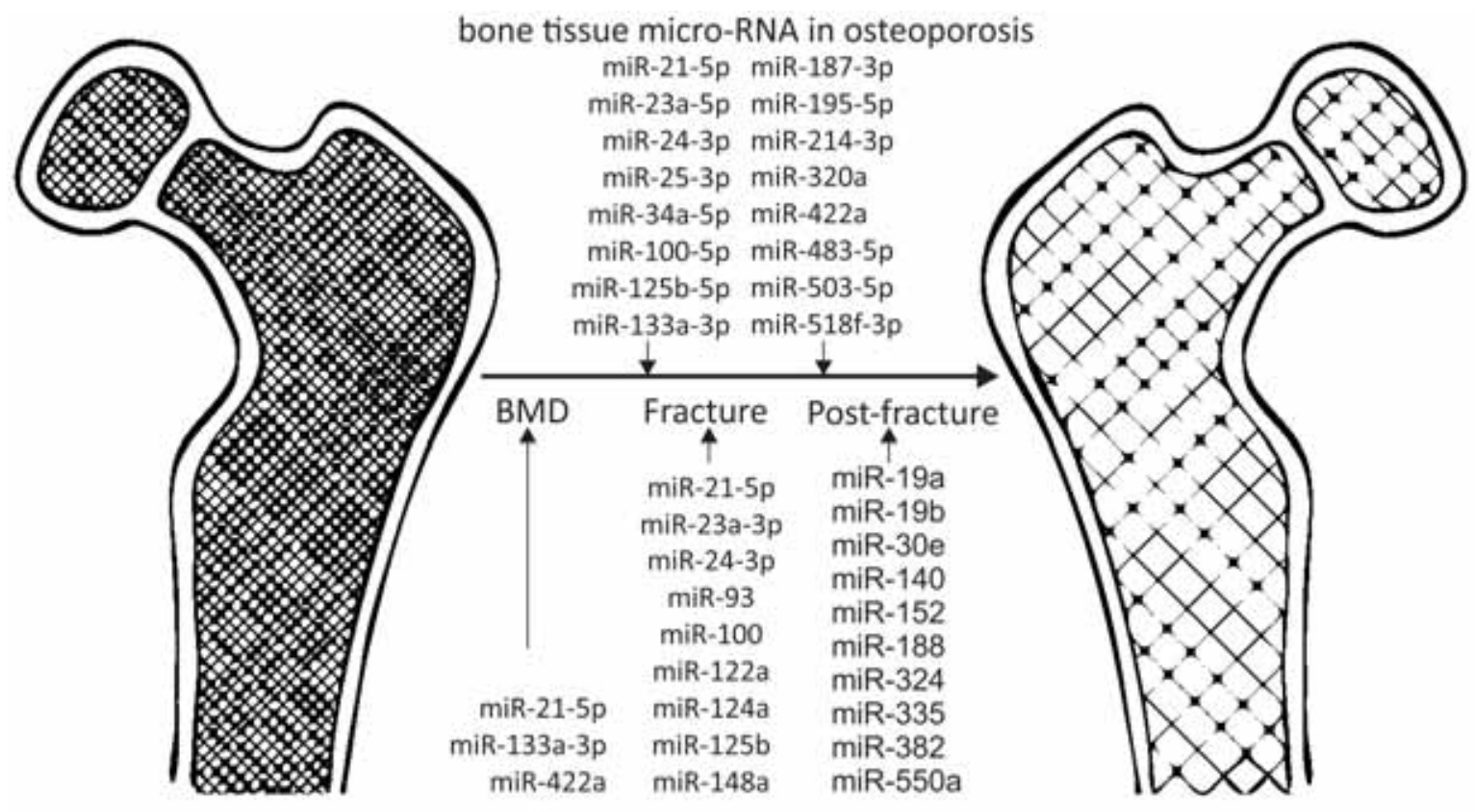

circulating micro-RNA in osteoporosis

FIGURE 2. Micro-RNA with role in osteoporosis etiopathogenesis.

The horizontal arrow represents the progression of bone demineralization and osteoporotic trabecular microarhitectural alterations. The two top columns contain subtypes of bone micro-RNA associated with osteoporosis. The three columns below the horizontal arrow contain the subtypes of plasma circulating micro-RNA, associated with osteoporosis. miR-21-5p, miR-133a-3p, miR-422a correlates with BMD, miR-21-5p, miR-23a-3p, miR-24-3p, miR-93, miR- miR-124a, miR-125b, miR-148a correlate with recent fractures and miR-19a, miR-19b, miR-30e, miR-140, miR-382, miR-550a correlate with old fractures (17).

Fig. 2 presents the micro-RNA subtypes that have a role in the osteoporotic etiopathogenesis.

\section{Noncoding RNA in the pathogenesis of periodontal disease}

Regarding the role of non-coding RNA in the pathogenesis of periodontal disease, it has been shown that mi-RNA exhibits higher concentrations in patients with periodontal disease (PD) compared to the healthy population. The most overexpressed mi-RNA subtypes are miR-150, miR-223, and miR-200b and the most under-expressed are miR-379, miR-199a-5p, miR-214. Overexpression of mi-RNA is generally associated with inflammatory diseases, cancer, urological disorders, and osteoporosis (18-22).

$\mathrm{Na}$ et al. and Lee et al., analyzing micro-RNAs in periodontally healthy patients and in patients with $\mathrm{PD}$, mention the increase in miR-19b, miR-30a, miR-23a, miR-181b, miR-7a, miR-128, miR-34a, miR-301a and miR-381 in the inflamed gum. They also report lower concentrations of miR-211, miR372 , miR-656 $(23,24)$.

Ogata et al. report higher concentrations of miR200b, miR-223, miR-150 and lower concentrations of miR-199a, miR-214 (25).
Xie et al. mention higher concentrations of miR146a si -146b, miR-223, miR-17, miR-203, miR19a, miR-142, miR-126, miR-20a in the inflamed marginal periodontium and lower concentrations of miR-205, miR-155 (26).

Stoeckin-Wasmer et al. report higher concentrations of miR-3917, 486, 223, 451 and lower concentrations of miR-205, 210, 203, 141, 1260, 1246 (27).

Lee et al. and Sugatani et al. mention the role of miR-223 in osteoclastic differentiation. Lowering the miR-223 concentration reduces the osteoclastic differentiation, while increasing the miR-223 concentration associates with osteoclastic formation. miR-223 associates directly with the inflammatory syndrome in the marginal periodontium in PD, participates in the alveolar bone loss and is presently studied as a possible epigenetic marker for PD $(24,28)$.

Fig. 3 presents the micro-RNA subtypes that have a role in the etiopathogenesis of PD.

miR-223 is a subtype of micro-RNA with a role in the differentiation of adipocytes and osteoclasts from HSC (hematopoietic stem cell). miR-223 decreases the expression of NFIA, an inhibitor of osteoclastic differentiation. Decreasing NFIA results 


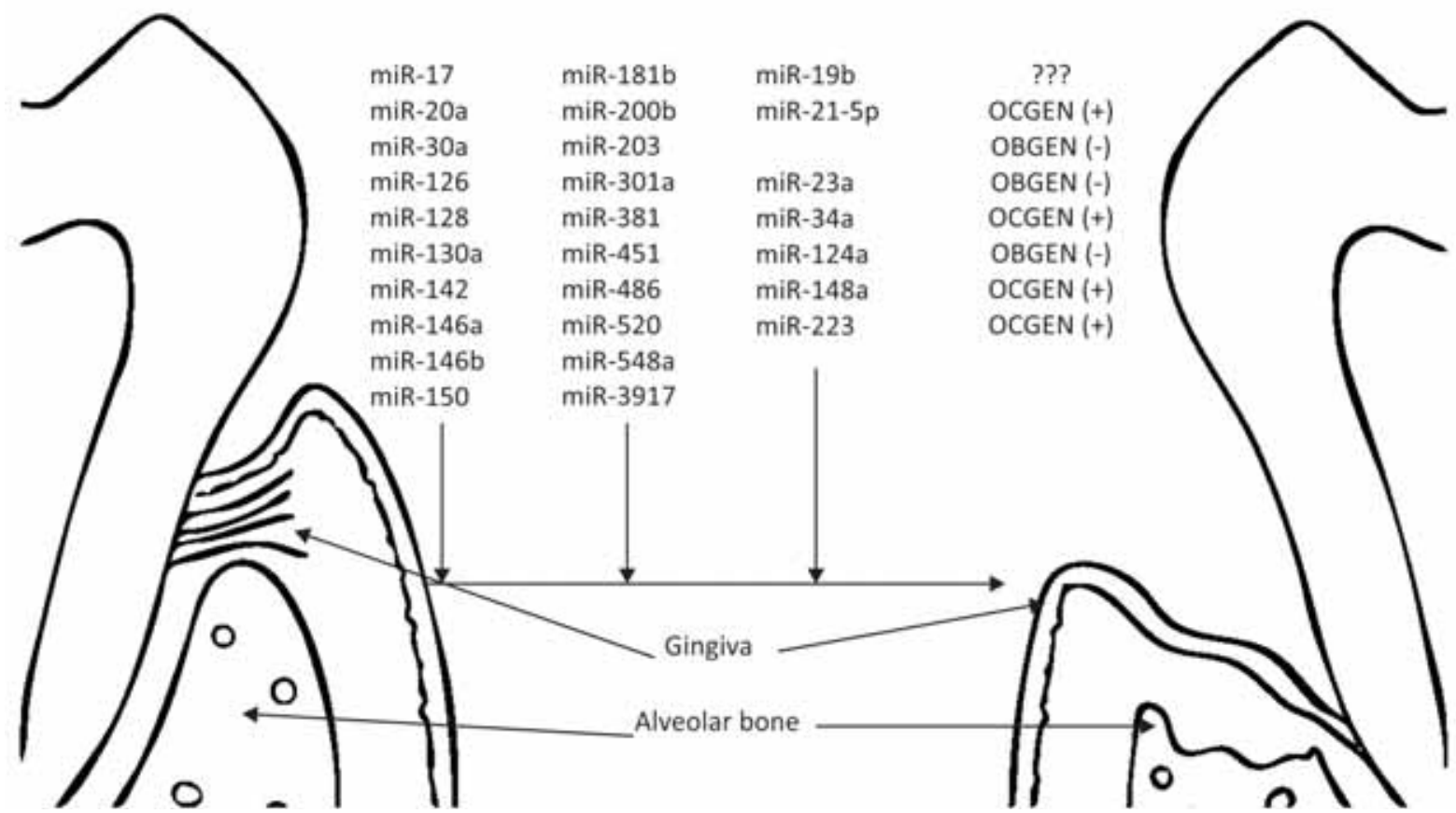

FIGURE 3. Micro-RNA with a role in PD epigenetics.

The two columns on the left side of the image show subtypes of micro-RNA that have been found inconstantly in the marginal periodontium in PD. The third column represents the representative micro-RNA subtypes for $P D$. Of these, miR-223 is the most constant overexpressed subtype mentioned in the literature. OCGEN-osteoclastogenesis; OBGEN-osteoblastogenesis; (+) stimulates; (-) inhibits (23-31).

in increased M-CSFR expression (M-CSF receptor) and implicitly increased M-CSF (macrophage colony-stimulating factor) activity, a stimulating factor for osteoclastogenesis. M'Baya-Moutoula E et al. Mention that miR-223 acts on three important factors in the osteoclastogenesis process: OPG (osteoprotegerin), TNF- $\alpha$ and NFKB. miR-223 decreases OPG expression and increases expression of TNF- $\alpha$ and NFkB. They mention that experimental therapy with miR-223 inhibitors results in increased expression of OPG, respectively decreased expression of TNF- $\alpha$ and NFkB, inhibiting osteoclastogenesis. Increased expression of miR-223 is strongly associated with increased bone loss. Xie et al. mention the inhibitory role of miR-223 in osteoblastogenesis. miR-223 reduces osteoblastic differentiation from MSC and inhibits FGFR2 (Fibroblast growth factor receptor 2), a modulator of osteoblastic differentiation. Activation of FGFR2 is associated with osteoblastic differentiation by increasing RUNX2 expression. miR-223 indirectly decreases RUNX2 expression and osteoblastogenesis (32-34).

\section{COMMON EPIGENETIC ELEMENTS}

miR-23a is a micro-RNA subtype associated with periodontal inflammatory syndrome. miR-23a in- hibits osteoblastogenesis by inhibiting RUNX2 in the first MSC (mesenchymal stem cells) differentiating phase to preosteoblast. Also, Seelinger et al., quoted by Ostanek, mention the association between miR-23a and osteoporosis. They note its bone overexpression in osteoporotic patients. It is a both tissue and plasma marker for osteoporosis and correlates directly with recent bone fractures $(17,23,24,35)$.

miR-19b is a micro-RNA subtype associated with periodontal inflammatory syndrome. Kocijan et al., quoted by Ostanek, frames miR-19b as a post-fracture circulatory marker in osteoporotic patients. Its plasma concentration is inversely proportional to $\operatorname{BMD}(17,23,24,36)$.

miR-34a is another micro-RNA subtype that associates with gingival inflammation. It promotes osteoclastogenesis and is a tissue marker associated with osteoporosis $(17,23,24,37)$.

Du A et al., quoted by Irwandi, mention the increase in miR-21-5p expression in the periodontal ligament treated with $P$. gingivalis lipopolysaccharide. miR-21-5p is referred to by Seelinger as having increased bone expression in osteoporotic patients. It is a tissue and plasma marker associated with osteoporosis, which correlates with recent fractures and $\operatorname{BMD}(17,29,35,38-40)$. 
In a recent meta-analysis, Kagiya $\mathrm{T}$ et al. mention higher concentrations of miR-21-5p, miR-124a and miR-148a in the marginal periodontium in PD. miR21-5p and miR-148a are known to be promoters of osteoclastogenesis. At the same time, these three types of micro-RNA are osteoporotic markers as follows: miR-21-5p is a tissue and plasma marker, is well expressed in bone in osteoporosis and is directly associated with BMD; miR-124a is an osteoporosis plasma marker and is associated with recent fractures; miR-148a is a osteoporosis plasma marker and is associated with recent fractures $(35,39-41)$.

\section{CONCLUSIONS}

The role of micro-RNA in the pathogenesis of osteoporosis and periodontal disease is little known. Studies in the literature that research this subject are just beginning.

There is no clear consensus yet on epigenetic changes in micro-RNA in osteoporosis or in periodontal disease. Studies analyzing mi-RNA changes report different and sometimes contradictory variations of different subtypes. This may be due, on the

\section{REFERENCES}

1. Marini F., Cianferotti L., Brandi M.L. Epigenetic Mechanisms in Bone Biology and Osteoporosis: Can They Drive Therapeutic Choices? Int J Mol Sci. 2016;17(8). pii: E1329

2. Hsu Y.H., Kiel D.P. Clinical review: Genome-wide association studies of skeletal phenotypes: What we have learned and where we are headed. J. Clin. Endocrinol. Metab. 2012; 97:1958-1977

3. Ghayor C., Weber F.E. Epigenetic Regulation of Bone Remodeling and Its Impacts in Osteoporosis. Int J Mol Sci. 2016; 17(9). pii: E1446

4. Consortium E.P. The ENCODE (ENCyclopedia Of DNA Elements) Project. Science 2004; 306:636-40.

5. lyer M.K., Niknafs Y.S. Malik R. et al. The landscape of long noncoding RNAs in the human transcriptome. Nat. Genet. 2015; 47:199-208.

6. Mercer T.R., Dinger M.E., Mattick J.S. Long non-coding RNAs: Insights into functions. Nat. Rev. Genet. 2009: 10: 155-9.

7. Zhu L., Xu P.C. Downregulated LncRNA-ANCR promotes osteoblast differentiation by targeting EZH2 and regulating Runx2 expression. Biochem. Biophys. Res. Commun. 2013; 432:612-17.

8. Tong X., Gu P.C., Xu S.Z. et al. Long non-coding RNA-DANCR in human circulating monocytes: A potential biomarker associated with postmenopausal osteoporosis. Biosci. Biotechnol. Biochem. 2015; 79:732-7.

9. Islam R., Bae H.S., Yoon W.J. et al. Pin1 regulates osteoclast fusion through suppression of the master regulator of cell fusion DC-STAMP. J. Cell. Physiol. 2014; 229:2166-74.

10. Kim K.J., Yeon J.T., Choi S.W. et al. Decursin inhibits osteoclastogenesis by downregulating NFATc1 and blocking fusion of pre-osteoclasts. Bone 2015; 81:208-16.

11. Dou C., Zhang C., Kang F. et al. MiR-7b directly targets DCSTAMP causing suppression of NFATc1 and c-Fos signaling during one hand, to age and gender variables in the groups analyzed and, on the other hand, to the lack of clear criteria for classifying gingival inflammation. Other causes may be the variability of exposure to environmental factors, especially at the site of harvesting, differences in the systemic and gingival bone harvesting method, and the large variability of the cell types that have been analyzed, particularly in the periodontium.

However, we have identified in the literature 6 micro-RNA subtypes that are recognized as plasma or tissue markers in systemic osteoporosis that correlate with systemic bone density, old or recent fractures, and whose concentration is increased in the periodontium in periodontal disease.

Interaction between environmental factors and genetic factors leads to epigenetic changes. Over- or under-expression of certain micro-RNA subtypes causes changes in bone homeostasis with disruption of the balance between resorption and apposition. Systemic epigenetic bone changes appear to occur in the alveolar bone, influencing inflammatory gingival response and progression of the infectious process.

Conflict of interest: none declared Financial support: none declared

osteoclast fusion and differentiation. Biochim. Biophys. Acta 2014; 1839:1084-96.

12. Weilner S., Skalicky S., Salzer B. et al. Differentially circulating miRNAs after recent osteoporotic fractures can influence osteogenic differentiation. Bone 2015; 79:43-51.

13. Seeliger C., Karpinski K., Haug A.T. et al. Five freely circulating miRNAs and bone tissue miRNAs are associated with osteoporotic fractures. J. Bone Miner. Res. 2014; 29:1718-28.

14. Guo D.W., Han Y.X., Cong L. et al. Resveratrol prevents osteoporosis in ovariectomized rats by regulating microRNA-3383p. Mol. Med. Rep. 2015; 12:2098-2106.

15. Khazaei S., Khazaei M., Kazemi S. et al. Resveratrol as a supplemental treatment for periodontitis. Dental Research Journal. 2012; 9(5):655-657.

16. Zupancic S., Baumgartner S., Lavric Z. et al. Local delivery of resveratrol using polycaprolactone nanofibers for treatment of periodontal disease. Journal of Drug Delivery Science and Technology. 2015; 30:408-16

17. Ostanek B., Kranjc T., Lovsin et al. Epigenetic Mechanisms in Osteoporosis. In: Moskalev A, Vaiserman A. Epigenetics of Aging and Longevity. Academic Press, 2018:365-388

18. Srinivasan P.C. The emerging role of epigenetics in the pathogenesis of periodontitis - A review. South African Dental Journal. 2016; 71(1):26-33

19. Xie Y.F., Shu R., Jiang S.Y. et al. Comparison of microRNA profiles of human periodontal diseased and healthy gingival tissues. Int $\mathrm{J}$ Oral Sci. 2011; 3:125-34.

20. Lee Y.H., Hee S.N., Jeong S.Y. et al. Comparison of inflammatory microRNA expression in healthy and periodontitis tissues. Biocell. $2011 ; 35: 42-9$. 
21. Stoecklin-Wasmer C., Guarnieri P., Celenti R. et al. MicroRNAs and their target genes in gingival tissues. J Dent Res. 2012; 91:934-40.

22. Ogata Y., Matsui S., Kato A. et al. Original MicroRNA expression in inflamed and non-inflamed gingival tissues from Japanese patients. Journal of Oral Science 2014; 56, (4):253-60.

23. Na H.S., Park M.H., Song Y.R. et al. Elevated miR-128 in periodontitis mitigates tumor necrosis factor-alpha response via P38 signaling pathway in macrophages. J Periodontol. 2016 Sep; 87(9):e173-82

24. Lee Y.H., Na H.S., Jeong S.Y. et al. Comparison of inflammatory microRNA expression in healthy and periodontitis tissues. Biocell. 2011; 35(2):43-9.

25. Ogata Y., Matsui S., Kato A. et al. MicroRNA expression in inflamed and noninflamed gingival tissues from Japanese patients. Journal of Oral Science. 2014:56(4):253-60.

26. Xie Y.F., Shu R., Jiang S.Y. et al. Comparison of microRNA profiles of human periodontal diseased and healthy gingival tissues. International Journal of Oral Science. 2011:3(3):125-34.

27. Stoecklin-Wasmer C., Guarnieri P., Celenti R. et al. MicroRNAs and their target genes in gingival tissues. Journal of Dental Research, 2012; 91(10):934-40.

28. Sugatani T., Hruska K.A. Impaired micro-RNA pathways diminish osteoclast differentiation and function. Journal of Biological Chemistry. 2009; 284(7):4667-78.

29. Irwandi R.A., Vacharaksa A. The role of microRNA in periodontal tissue: A review of the literature. Arch Oral Biol. 2016; 72:66-74. doi: 10.1016/j.archoralbio.2016.08.014. Epub 2016 Aug 13.

30. Wei F., Yang S., Guo Q. MicroRNA-21 regulates Osteogenic Differentiation of Periodontal Ligament Stem Cells by targeting Smad5. Sci Rep. 2017;7(1):16608

31. Qadir A.S., Um S., Lee H. miR-124 negatively regulates osteogenic differentiation and in vivo bone formation of mesenchymal stem cells. J Cell Biochem. 2015;116(5):730-42

32. Lian J.B., Stein G.S., van Wijnen A.J. et al. MicroRNA control of bone formation and homeostasis. Nature reviews Endocrinology. 2012; 8(4):212-227. doi:10.1038/nrendo.2011.234.
33. M‘Baya-Moutoula E., Louvet L., Metzinger-Le Meuth V. et al. High inorganic phosphate concentration inhibits osteoclastogenesis by modulating miR-223. Biochim Biophys Acta. 2015; 1852(10 Pt A):2202-12. doi: 10.1016/j.bbadis.2015.08.003.

34. Xie Y., Zhang L., Gao Y. et al. The Multiple Roles of Microrna-223 in Regulating Bone Metabolism. Molecules. 2015; 20(10):19433-48

35. Seeliger C., Karpinski K., Haug A.T. et al. Five freely circulating miRNAs and bone tissue miRNAs are associated with osteoporotic fractures. J Bone Miner Res 2014; 29(8):1718-28.

36. Kocijan R., Muschitz C., Geiger E. et al. Circulating microRNA signatures in patients with idiopathic and postmenopausal osteoporosis and fragility fractures. J Clin Endocrinol Metab 2016; 101(11):4125-34.a

37. Krzeszinski J.Y., Wei W., Huynh H. et al. miR-34a blocks osteoporosis and bone metastasis by inhibiting osteoclastogenesis and Tgif2. Nature 2014; 512(7515):431-5.

38. Du A., Zhao S., Wan L.Y. et al. MicroRNA expression profile of human periodontal ligament cells under the influence of Porphyromonas gingivalis LPS. J Cell Mol Med. 2016; 20:1329-1338.

39. Kagiya T. MicroRNAs: Potential Biomarkers and Therapeutic Targets for Alveolar Bone Loss in Periodontal Disease. Int J Mol Sci. 2016 Aug 11;17(8). pii: E1317. doi: 10.3390/ijms17081317.

40. Li H., Wang Z., Fu Q. et al. Plasma miRNA levels correlate with sensitivity to bone mineral density in postmenopausal osteoporosis patients. Biomarkers 2014;19(7):553-6.

41. Heilmeier U., Hackl M., Skalicky S. et al. Serum miRNA signatures are indicative of skeletal fractures in postmenopausal women with and without type 2 diabetes and influence osteogenic and adipogenic differentiation of adipose tissue-derived mesenchymal stem cells in vitro. J Bone Miner Res 2016; 31(12):2173-92. 